\title{
Psychiatric Symptoms and Benzodiazepine Use Mask a Patent Foramen Ovale-Related Stroke: A Cautionary Tale
}

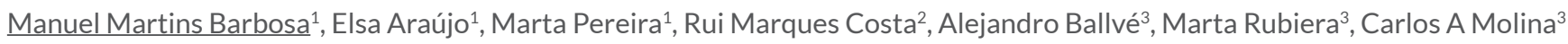 \\ ${ }^{1}$ Unidade Local Saude Alto Minho, EPE, Portugal \\ ${ }^{2}$ Centro Hospitalar de Lisboa Ocidental, EPE, Portugal \\ ${ }^{3}$ Stroke Unit, Neurology Department, Hospital Universitari Vall d'Hebron, Barcelona, Spain
}

Doi: 10.12890/2019_001120 - European Journal of Case Reports in Internal Medicine - ๑ EFIM 2019

Received: $14 / 04 / 2019$

Accepted: 08/05/2019

Published: 05/07/2019

How to cite this article: Barbosa Martins M, Araújo E, Pereira M, Marques Costa P, Ballvé A, Rubiera M, Molina CA. Psychiatric symptoms and benzodiazepine use mask a patent foramen ovale-related stroke: a cautionary tale. EJCRIM 2019;6: doi:10.12890/2019_001120.

Conflicts of Interests: The Authors declare that there are no competing interests.

This article is licensed under a Commons Attribution Non-Commercial 4.0 License

\section{ABSTRACT}

Neurological and psychiatric diseases often present with overlapping features. Patients are sometimes seen by internal medicine, neurology and psychiatry clinicians and time can be important for determining whether a condition is organic or not.

The authors present the case of a 31-year-old woman admitted to the emergency department with altered mental status and incomprehensible speech. She had a previous history of anxiety and smoking and was under therapy with benzodiazepines and combined hormonal contraceptives.

After an acute neurological disorder was ruled out, the patient was transferred to the psychiatry department, where she was re-evaluated after 12 hours of observation and benzodiazepine washout. As speech impairment and confusion were still present, she was transferred to the Stroke Unit. A bilateral thalamic lesion containing a small focal haemorrhage and a moderate grade right-to-left shunt were identified. A patent foramen ovale was confirmed leading to the final diagnosis of acute ischaemic infarction secondary to a paradoxical embolism.

Medical therapy was preferred over percutaneous closure. Risk factors were removed (smoking and hormonal contraceptives), and after the patient recovered completely acetylsalicylic acid $100 \mathrm{mg}$ daily was initiated.

\section{LEARNING POINTS}

- Psychiatric symptoms and medication often mask organic conditions, so observation and re-evaluation are key.

- There are new guidelines for the treatment of patent foramen ovale-related strokes, but not all patients fit the guidelines.

- A relationship between stroke in the young adult, smoking and hormonal contraceptives should always be considered.

\section{KEYWORDS}

Psychiatric mimics, patent foramen ovale, PFO closure, PFO medical therapy, stroke in a young adult

\section{CASE DESCRIPTION}

A 31-year-old female patient with a history of anxiety and depression was transported to the emergency department (ED) after activation of a stroke code alert. She had a previous history of smoking and anxiety, and was under regular therapy with alprazolam and fluoxetine. She had recently started oral contraceptives and was an occasional smoker.

More than 8 hours before her arrival at the ED, a familial altercation had taken place. The patient then locked herself in a bathroom, which was the last time she was seen without symptoms. Hours later she was found extremely sleepy and with incoherent speech. It was unclear at that time if she had taken an overdose of her usual medical therapy. Emergency medical system (EMS) services were called and a stroke code alert was activated. 
Upon arrival at hospital, the patient was transferred to the CT suite were she was observed. She was extremely sleepy, required repeated stimulation to attend to her surroundings, and had her eyes closed for most of the time. She was partially disoriented in time, but not in space. Her speech was poor and incoherent and she could not perform most of the complex tasks requested. A possible mild central facial palsy was noted. Gaze and visual fields were normal. She had no motor or sensory deficits. She presented no signs of aphasia and osteotendinous reflexes were normal. She showed no positive signs of meningeal irritation. The National Institutes of Health Stroke Scale (NIHSS) score was 4.

Brain CT scans with arterial and perfusion studies were performed and ruled out acute ischaemic or haemorrhagic lesions. Due to the long interval since the patient was last seen in a normal state and the low NIHSS score, medical thrombolysis was not initiated.

Flumazenil was administered in order to reverse benzodiazepine intoxication, with no effect.

A lumbar puncture (LP) was performed to rule out central nervous system infection. Cerebrospinal fluid showed normal glucose levels (73 $\mathrm{mg} / \mathrm{dl}$ ), no white blood cells ( 3 cells/ $/ \mathrm{l}$ ) and $65 \mathrm{mg} / \mathrm{dl}$ of proteins, interpreted as the consequence of a traumatic LP (over 12,000 red blood cells/ $\mu \mathrm{l})$.

The remaining physical examination was normal, with a blood pressure of $109 / 60 \mathrm{mmHg}$, a pulse of 54 bpm, peripheral $\mathrm{O}_{2}$ saturation of $100 \%$ and an axillary temperature of $36.4^{\circ} \mathrm{C}$. Heart sounds were normal, respiratory sounds were symmetrical and distal perfusion was preserved.

A sinus rhythm was seen on electrocardiogram (EKG). Blood tests were unremarkable as regards the haemogram, coagulation and acutephase reactants (including erythrocyte sedimentation rate) and thyroid hormone levels were normal. Urine analysis was positive for benzodiazepines. An electroencephalogram (EEG) was performed, ruling out epileptic foci.

The patient was then transferred to the psychiatry department for further evaluation of a suspected conduct disorder. Her family was restless, repeating countless times that they had never seen her in that state. After 12 hours of observation and benzodiazepine washout, the patient had not improved and so was admitted to the Stroke Unit for further study and care after a second consultation with the neurology department.

Brain MRI was performed and showed a bilateral thalamic lesion on diffusion, ADC and FLAIR sequences, mostly in the right hemisphere with a small focal haemorrhage inside (Figs. 1-3). Anti-thrombotic therapy was postponed due to the haemorrhagic focus.
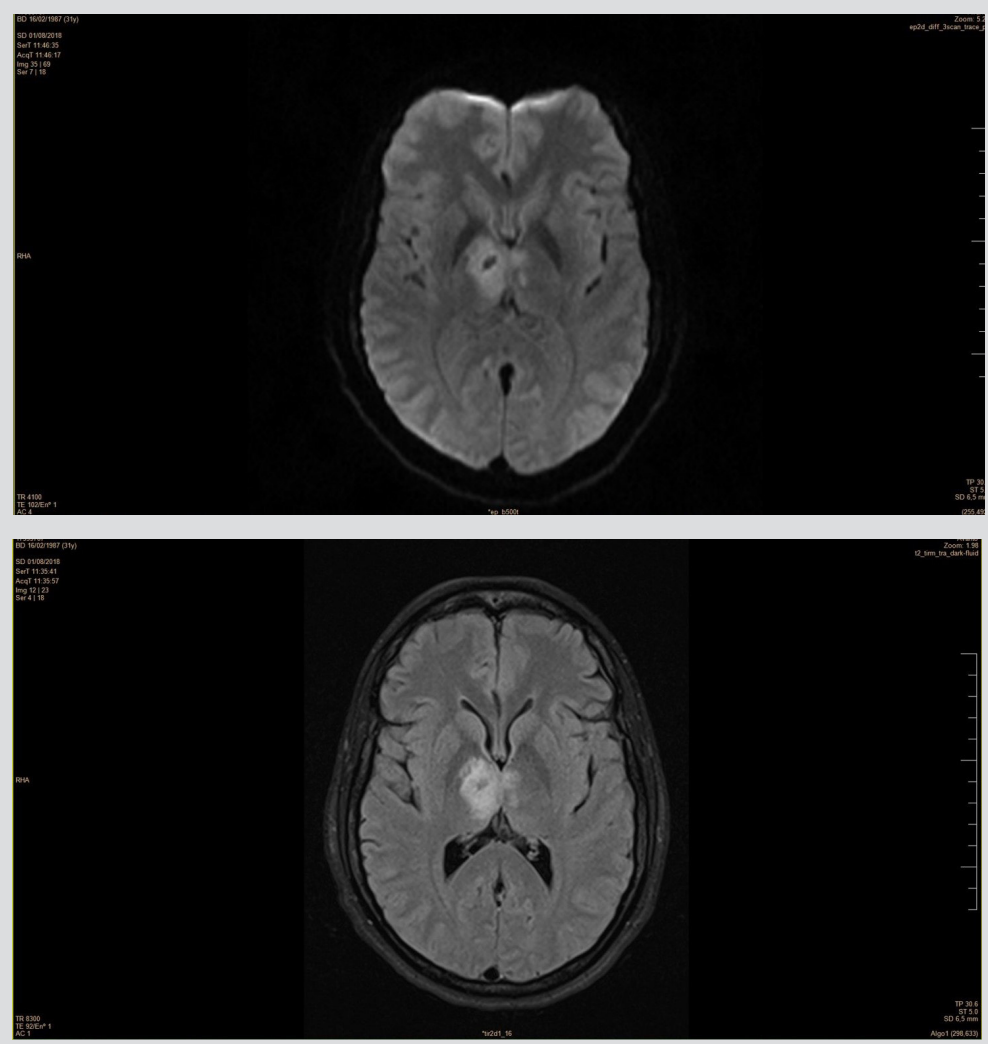

Figure 1. Diffusion sequence showing a bilateral thalamic lesion mostly on the right hemisphere

Figure 2. FLAIR sequence showing a bilateral thalamic lesion mostly on the right hemisphere 
The patient underwent aetiological study with echocardiogram and transcranial Doppler (TCD) ultrasonography that revealed an atrial septal aneurysm and a moderate right-to-left shunt with 12 micro-embolic signals (MES) after Valsalva manoeuvre (VM). The presence of a patent foramen ovale (PFO) was confirmed with transoesophageal echocardiography (TEE) the following day, and the final diagnosis of acute ischaemic infarction secondary to a paradoxical embolism was made.

To rule out other sources of emboli, the patient underwent a lower limb Doppler study that excluded deep venous thrombosis (DVT). Thrombophilia testing for protein C, protein S, antithrombin III, lupus anticoagulant, anticardiolipin antibodies, lupus anticoagulant and antinuclear antibodies was negative.

The patient's neurological condition improved over the following days and her NIHSS score dropped to zero. A head CT scan performed 1 week after the event showed no progression or new lesions compared with the baseline MRI.

The case was discussed with the cardiology department regarding medical treatment versus PFO closure. The final decision was to start anti-thrombotic medication with acetylsalicylic acid (AAS) $100 \mathrm{mg}$ daily. Oral contraceptives were stopped.

The patient was discharged home 7 days after admission with no deficits. She was referred to follow-up outpatient appointments in the psychiatry, internal medicine and neurology departments.

\section{DISCUSSION}

This case raises two important points. Firstly, it can be difficult to identify a stroke in a patient with other factors that suggest a psychiatric diagnosis, so patients need to be re-evaluated frequently. Psychiatric conditions and medication can mask other diseases. It is important to be aware of features which might suggest other diagnoses and support further investigation. New symptoms, thorough review of medications and side effects, and family reports of unusual behaviour should be considered by the physicians as warning flags ${ }^{[1]}$.

Secondly, current medical evidence regarding treatment of a recently diagnosed PFO and a moderate grade shunt should be reviewed. In late 2017, the results of trials such as CLOSURE and gore-REDUCE, demonstrated, for the first time, strong evidence that favoured PFO closure in selected patients rather than medical therapy ${ }^{[2]}$. The best suited candidates were younger, had moderate-to-severe PFO, septal aneurysm and a diagnosis of cryptogenic stroke after other aetiologies had been excluded ${ }^{[3]}$. A meta-analysis of these trials showed that, in this population, recurrence rate was lower (0.68\% per year vs. $1.16 \%$ per year) but the risk of atrial fibrillation was increased (1.38\% per year vs. $0.21 \%$ per year). Most current guidelines still do not recommend PFO closure, but the growing evidence suggests that an update is required ${ }^{[4]}$.

On the one hand, our patient seemed a good candidate for PFO percutaneous closure after the completion of all investigations. However, on the other hand, there were important risk factors that could have contributed to the embolic event and had to be accounted for: the patient was a smoker and had recently started hormonal contraceptives. Therefore, weighing risks versus benefits from the procedure, the final decision was to start anti-thrombotic therapy as secondary prevention with AAS and remove the well-known risk factors, thus reducing the risk of recurrence.

\section{REFERENCES}

1. Knight SR, Mallory MNS, Huecker MBA. Medical mimics of psychiatric conditions, part 1. Emerg Med 2016:48(5):202-2011.

2. Mojadidi MK, Zaman M, Elgendy IY, et al. Cryptogenic stroke and patent foramen ovale. J Am Coll Cardiol 2018;71(9):1035-1043.

3. Singh HS, Katchi F, Naidu SS. PFO closure for cryptogenic stroke - a review and clinical treatment algorithm. Cardiol Rev 2017;25:147-157.

4. Ahmad Y, Howard JP, Arnold A, Shin MS, Cook C, et al. Patent foramen ovale closure vs. medical therapy for cryptogenic stroke: a meta-analysis of randomized controlled trials. Eur Heart J 2018;39(18):1638-1649. 